\title{
INGESTION DES MICROFILAIRES PAR LE VECTEUR DANS LE CAS DES BASSES MICROFILAREMIES (1)
}

\author{
G. PETIT* et G. PICHON**
}

RÉSUMÉ. L'étude de l'ingestion des microfilaires de Dipetalonema dessetae par Aedes aegypti permet de tirer deux conclusions.

Quelle que soit la microfilarémie :

- il n'y a pas de concentration des microfilaires lors de l'ingestion par l'Aedes.

- les microfilaires sont réparties d'une façon quasi-homogène dans le sang de l'hôte vertébré accessible à la piqûre par le vecteur.

Une telle répartition des microfilaires dans le sang cutané de l'hôte donne le maximum de chances à un moustique de s'infecter lors du repas sanguin.

Dans le couple Wuchereria bancrofti - Aedes polynesiensis, le pouvoir infectant des individus peu microfilariens paraît résulter du phénomène de limitation.

\section{Intake of microfilariae by the vector in the case of a low microfilareamia.}

SUMMARY. The study of the intake of microfilariae of Dipetalonema dessetae by Aedes aegypti leads to two conclusions with respect to the microfilaraemia :

- there is no concentration of microfilariae in the uptake of blood by Aedes.

- the microfilariae are nearly homogeneously distributed in the vertebrate host blood available to the vector for feeding.

Such a distribution of microfilariae in the cutaneous blood supply of the host gives the maximum chance for a mosquito to become infected when taking a blood meal.

In the pair Wuchereria bancrofti - Aedes polynesiensis the capacity of individuals with a low microfilaraemia to transmit infections seems to be related to a limitation phenomenon.

\section{Introduction}

Les traitements de masse à la diethylcarbamazine des foyers de filariose lymphatique humaine laissent persister de très faibles taux de microfilaires dans le sang de certains individus traités; ceux-ci semblent capables d'assurer le maintien du foyer.

* Laboratoire de Zoologie (Vers), associé au CNRS, Muséum national d'Histoire naturelle, 61, rue Buffon F 75231 Paris Cedex 05.

** ORSTOM, 50-74 route d'Aulnay, F 93140 Bondy.

Adresse actuelle : Laboratoire d'épidémiologie de la D.D.A.S.S., 8 rue J. Chatel, 97400 St-Denis (La Réunion).

(I) Ce travail a été effectué grâce à une subvention de l'Organisation Mondiale de la Santé. Accepté le II septembre r 981 . 
Plusieurs auteurs ont envisagé l'existence d'une concentration des microfilaires dans le cas des faibles microfilarémies pour expliquer ce phénomène (Bryan J. H. et Southgate B. A., 1976 ; Samarawickrema W. A. et al., 1979).

A l'aide de la filaire de rongeur Dipetalonema dessetae Bain, 1973, transmise expérimentalement par Aedes aegypti, nous recherchons l'existence d'une telle concentration en comparant l'ingestion des microfilaires dans le cas des basses microfilarémies et des fortes microfilarémies. Deux aspects principaux de l'ingestion semblent importants à étudier :

- le nombre moyen de microfilaires ingérées : comparaison entre le nombre moyen attendu et le nombre moyen observé ;

- la distribution des microfilaires dans le sang cutané de l'hôte vertébré et son influence sur le pourcentage de moustiques qui s'infecte lors de l'ingestion.

Les résultats de cinq expériences sont analysés (tableaux $I$ et $I I$ ). Les conditions expérimentales sont les suivantes :

- Obtention des fortes microfilarémies par injection sous-cutanée de 50 stades infestants de $D$. dessetae à des Proechimys sains (expériences 21 GK, 35 GK et 37 GK).

- Obtention des faibles microfilarémies par injection intrapéritonéale de sang d'un Proechimys très infesté à des Proechimys sains, (expériences 39 GK et $44 \mathrm{GK}$ ).

- Mesure de la microfilarémie sur un prélèvement de $0,1 \mathrm{ml}$ de sang au sinus de l'œil du rongeur avant chaque gorgement (dans le tableau $I$, la microfilarémie mesurée est rapportée à un volume de $1 \mathrm{ml}$ de sang).

- Gorgement à réplétion des Aedes sur la peau abdominale du rongeur pendant le plateau diurne de microfilarémie maximale; seuls les moustiques de même taille ayant ingéré un volume maximum de sang sont disséqués pour dénombrer les microfilaires intrastomacales (les moustiques sont placés à $0^{\circ} \mathrm{C}$ juste après le gorgement pour éviter que les microfilaires passent dans l'hémocèle).

A - Nombre moyen de microfilaires ingérées : comparaison entre le nombre moyen attendu et le nombre moyen observé (Tableau I, colonnes 3, 4, 5)

- Le nombre moyen attendu de microfilaires ingérées par Aedes est calculé en fonction de la densité microfilarienne au sinus de l'œil en estimant que chaque Aedes ingère $4,5 \mathrm{~mm}^{3}$ de sang (Petit, sous presse).

- Dans le cas des fortes densités microfilariennes (expériences $21 \mathrm{GK}, 35 \mathrm{GK}, 37 \mathrm{GK}$ ), le rapport $\frac{\text { nombre moyen observé }}{\text { nombre moyen attendu }}$ varie entre 1,09 et 1,13; dans le cas des faibles densités microfilariennes, il est égal à 1,25 (expérience $39 \mathrm{GK}$ ) et 1,51 (expérience $44 \mathrm{GK})$.

- Ce rapport ne varie pas significativement en fonction de la densité microfilarienne et ne met donc pas en évidence un phénomène de concentration des microfilaires dans le cas des faibles microfilarémies étudiées ici. 


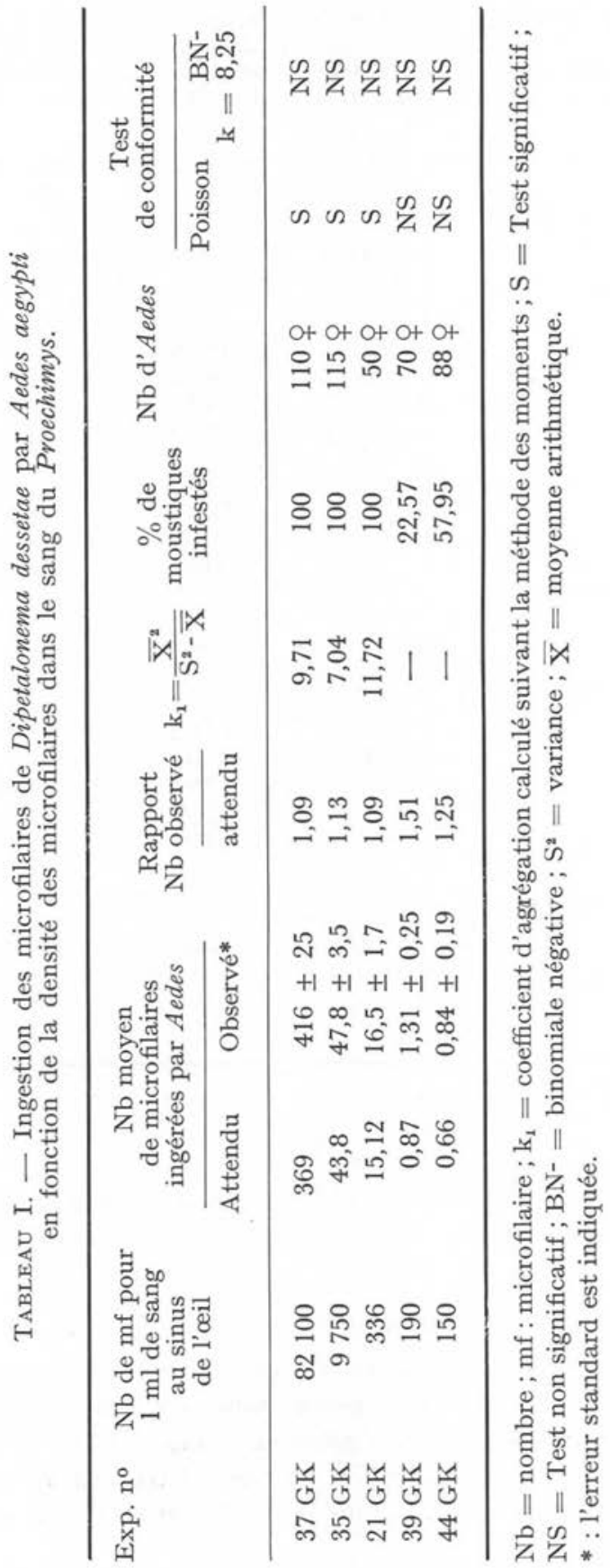


TABleau II. - Distribution observée des microfilaires ingérées par Aedes aegypti sur 2 Proechimys peu infestés par $D$. dessetae (expériences 39 GK et $44 \mathrm{GK}$ ) ; Comparaison aux fréquences calculées pour différents degrés d'agrégation et pour une même moyenne (les accolades indiquent les regroupements effectués pour les calculs de $\chi$ ).

\begin{tabular}{|c|c|c|c|c|c|}
\hline & \multirow{2}{*}{$\begin{array}{l}\text { Nb de mf } \\
\text { par Aedes }\end{array}$} & \multicolumn{4}{|c|}{ Nombre d'Aedes } \\
\hline & & Observés & $\mathrm{k}=1$ & $\mathrm{k}=8,25$ & Poisson \\
\hline $\begin{array}{c}\text { Exp. } \\
39 \text { GK } \\
\mathrm{N}=70\end{array}$ & $\begin{array}{l}0 \\
1 \\
2 \\
3 \\
4 \\
5\end{array}$ & $\left.\begin{array}{r}15 \\
29 \\
19 \\
5 \\
2\end{array}\right\}$ & $\begin{array}{r}30,25 \\
17,18 \\
9,75 \\
5,54 \\
3,15 \\
+\quad 4,13\end{array}$ & $\begin{array}{r}20,68 \\
23,44 \\
14,90 \\
6,99 \\
2,70 \\
+\quad 0,29\end{array}$ & $\begin{array}{r}18,81 \\
24,72 \\
16,24 \\
7,12 \\
2,34 \\
+\quad 0,78\end{array}$ \\
\hline$\chi^{2}$ & & & 28,43 & 7,82 & 3,02 \\
\hline $\mathrm{P}\left(\chi^{2}\right)$ & & & $10^{-5}$ & 0,10 & 0,55 \\
\hline $\begin{array}{l}\text { Test de } \\
\text { conformité }\end{array}$ & & & $\mathrm{S}$ & NS & NS \\
\hline $\begin{array}{c}\text { Exp. } \\
44 \mathrm{GK} \\
\mathrm{N}=88\end{array}$ & $\begin{array}{l}0 \\
1 \\
2 \\
3\end{array}$ & $\begin{array}{r}37 \\
34 \\
11 \\
6\end{array}$ & $\begin{array}{r}47,80 \\
21,84 \\
9,97 \\
4,56 \\
+\quad 3,67\end{array}$ & $\begin{array}{r}39,51 \\
30,15 \\
12,90 \\
4,08 \\
+\quad 1,36\end{array}$ & $\begin{array}{r}37,96 \\
31,92 \\
13,42 \\
3,76 \\
+\quad 0,95\end{array}$ \\
\hline$\chi^{2}$ & & & 82,04 & 0,99 & 0,95 \\
\hline $\mathrm{P}\left(\chi^{2}\right)$ & & & 0,01 & 0,80 & 0,81 \\
\hline $\begin{array}{l}\text { Test de } \\
\text { conformité }\end{array}$ & & & $\mathrm{S}$ & NS & NS \\
\hline
\end{tabular}

$\mathrm{N}$ : nombre d'Aedes, $\mathrm{P}$ : probabilité.

B - Distribution des Aedes en fonction du nombre de microfilaires ingérées : relation entre le coefficient d'agrégation $k$ et la densité microfilarienne dans le sang de l'hôte vertébré.

a - Analyse des distributions observées

- Dans le cas des fortes microfilarémies, ces distributions se rapprochent de la distribution homogène de type poissonnien (tableau I, colonne 6) (voir Petit, sous presse) ; en effet, le coefficient d'agrégation $\mathrm{k}$ a des valeurs élevées, qui peuvent dépasser 10. Pour les expériences $37 \mathrm{GK}, 35 \mathrm{GK}, 21 \mathrm{GK}$, où le coefficient $\mathrm{k}$ est calculable, la valeur commune de $\mathrm{k}$ la plus probable (méthode du maximum de vraisemblance) est 8,25 . 
- Dans le cas des faibles microfilarémies (expériences $39 \mathrm{GK}$ et $44 \mathrm{GK}$ ) (tableaux I et $I I$ ), le coefficient $\mathrm{k}$ n'est pas calculable, mais les distributions observées se rapprochent également du modèle poissonnien; en effet, le rapport $\frac{\mathrm{S}^{2}}{\overline{\mathrm{X}}}$ ne diffère pas significativement de 1 (Test de Bliss et Fisher, 1953). Ces deux distributions sont également compatibles avec une loi binomiale négative de coefficient $\mathrm{k}=8,25$.

- Lorsque chaque Aedes ingère un même volume de sang, la distribution des Aedes en fonction du nombre de microfilaires ingérées donne l'image de la distribution des microfilaires dans le sang de l'hôte accessible à la piqûre du vecteur. Nous pouvons donc conclure ici que la distribution des microfilaires dans le sang cutané du rongeur, de type quasi-homogène dans le cas des fortes microfilarémies, l'est aussi dans le cas des faibles microfilarémies. infestés.

$\mathrm{b}$ - Influence théorique du degré d'agrégation $\mathrm{k}$ sur le pourcentage d'Aedes

Grâce à la formule donnant la proportion d'individus négatifs d'une loi binomiale négative (Bliss et Fisher, 1953 ; cf. aussi Pichon et al., 1980b, équation 1, et Cabaret, sous presse), on peut calculer le pourcentage théorique $\mathrm{P}$ d'Aedes qui s'infectent, en fonction du nombre moyen $\overline{\mathrm{X}}$ de parasites ingérés et pour différentes valeurs du coefficient d'agrégation $\mathrm{k}: \mathrm{P}=\left[1-\left\{\frac{\mathrm{k}}{\mathrm{k}+\overline{\mathrm{X}}}\right\}^{\mathrm{k}}\right] \times 100$.

- Dans le cas des fortes moyennes de microfilaires ingérées (exemple de l'expérience $37 \mathrm{GK})$ :

si $\mathrm{k}=0,5, \quad 96,5 \%$ des Aedes sont infestés ;

si $\mathrm{k}=1, \quad 99,8 \% \quad-\quad-\quad$;

si $\mathrm{k}=8,25,100 \% \quad-\quad-$ (pourcentage observé $=100 \%$ ).

- Dans le cas des faibles moyennes de microfilaires ingérées (exemple de l'expérience $44 \mathrm{GK})$ :

si $\mathrm{k}=0,5, \quad 38,9 \%$ des Aedes sont infestés ;

si $\mathrm{k}=3, \quad 52,3 \% \quad-\quad-\quad$;

si $\mathrm{k}=8,25, \quad 55,1 \% \quad-\quad-\quad$;

si la distribution est poissonnienne, $56,8 \%$ des Aedes sont infestés ( $\%$ observé : $57,9)$.

Pour une telle moyenne de microfilaires ingérées, une distribution homogène des microfilaires permet d'infecter nettement plus de moustiques qu'une distribution surdispersée $(56,8 \%$ contre $38,9 \%$ quand $\mathrm{k}=0,5)$.

- Pour des moyennes de microfilaires ingérées encore plus basses, seule l'approche théorique est possible. En effet, plusieurs centaines de moustiques gorgés sont nécessaires pour avoir une bonne approche du nombre moyen de microfilaires ingérées et pour étudier les distributions; mais, la petite taille du rongeur (350 g à l'état adulte) ne permet pas une telle expérimentation.

Dans cette approche théorique, les chiffres obtenus sont les suivants, pour une moyenne de microfilaires ingérées par Aedes égale à 0,1 : 
si $\mathrm{k}=0,01, \quad 2,4 \%$ des Aedes sont infestés ;

si $\mathrm{k}=0,5, \quad 8,7 \% \quad-\quad-\quad$;

si $\mathrm{k}=8,25, \quad 9,5 \% \quad-\quad-\quad$;

si la distribution est poissonnienne, 9,5\% des Aedes sont infestés.

\section{Conclusion}

Pour les fortes densités microfilariennes, ainsi que pour les faibles densités que nous étudions particulièrement ici, la filaire $D$. dessetae se caractérise par :

- l'absence de tout phénomène de concentration des microfilaires lors de l'ingestion par Aedes aegypti;

- une distribution quasi-homogène des microfilaires dans le sang de l'hôte vertébré accessible à la piqûre par le vecteur.

Ce type de répartition des microfilaires s'oppose aux distributions surdispersées observées généralement, dont le paramètre d'agrégation $\mathrm{k}$ est voisin de 1 (loi géométrique : Pichon et al., 1980a). Celles-ci nous semblent résulter des variations de la quantité de sang ingéré par le vecteur (Petit, sous presse).

Une telle distribution des microfilaires dans le sang cutané de l'hôte vertébré donne le maximum de chances au vecteur de s'infecter lors de la piqûre.

Le phénomène de concentration envisagé par Bryan J. H. et Southgate B. A., 1976, Samarawickrema W. A. et al., 1979, paraît correspondre à l'expression du phénomène de limitation (Bain, 1971), qui existe dans le couple Wuchereria bancrofti - Aedes polynesiensis (Pichon, 1974a, b ; Prod'hon et al., 1980b); dans le couple $W$. bancrofti - Aedes samoanus, qui ne présente pas cette limitation, aucun phénomène de concentration n'est mis en évidence.

\section{BIBLIOGRAPHIE}

Barn O. : Transmission des Filarioses. Limitation des passages des microfilaires ingérées vers l'hémocèle du vecteur; interprétation. Ann. Parasitol. Hum. Comp., 1971, 46, 613-631.

Bliss C. I., Fisher R. A. : Fitting the negative binomial distribution to biological data. Biometrics, I953, 9, I76-200.

Bryan J. H., Southgate B. A. : Some observations in filariasis in Western Samoa after mass administration of diethylcarbamazine. Trans. roy. Soc. trop. Med. Hyg., 1976, 70, 39-48.

CABARET J. : Les critères de réceptivité à l'infestation par les Protostrongylidés chez les Mollusques. Étude comparée dans les conditions expérimentales et naturelles. Ann. Parasitol. Hum. Comp. (sous presse).

Petrit G. : Ingestion des microfilaires par le vecteur dans le cas d'une Filaire périodique Dipetalonema dessetae. Ann. Parasitol. Hum. Comp., (sous presse).

Pichon G. : Relations numériques entre le nombre de microfilaires ingérées et le nombre de parasites chez différents vecteurs naturels ou expérimentaux de filarioses. Cah. ORSTOM, sér. Ent. méd. Parasitol., 1974b, I2, I99-216.

PICHON G., PROD'HON J., R'rvinRE F. : Hétérogénéité de l'ingestion des parasites sanguicoles par leurs vecteurs : description quantitative et interprétation. C. R. Acad. Sc. Paris, I980a, 290, IOII-IOI3.

PICHON G., PROD'HON J., RIVIÈRE F. : Filarioses : surdispersion parasitaire et surinfection de l'hôte vertébré. Cah. ORSTOM, sér. Ent. méd. Parasitol., 1980b, 18, 24-47.

ProD'HON J., PICHON G., RIVIÈRE F. : Étude quantitative de la réduction parasitaire stomacale chez les vecteurs de filaires. Cah. ORSTOM, sér. Ent. méd. Parasitol, 1980b, 18, 13-25.

Samarawickrema W. A., Folasone, Kazuyoichimori, Cumings R. F. : Filariasis in Western Samoa. I. Transmission studies of Aedes (Stegomyia) polynesiensis (Marks) and Aedes (Finlaya) samoanus (Gruenberg) on carriers with different levels of Microfilaraemia of Wucheria bancrofti. WHO. VBC. $79.75 \mathrm{I}, \mathrm{r} 979,2 \mathrm{I}$. 